\title{
Phenotypic and molecular characterization of HA-MRSA in Taif hospitals, Saudi Arabia
}

\author{
Emad M Eed ${ }^{1,2}$, Mabrouk M Ghonaim ${ }^{1,2}$, Yousry M Hussein ${ }^{1,3}$, Taiser M Saber ${ }^{1,4}$, Amany S Khalifa ${ }^{5,6}$ \\ ${ }^{1}$ Medical Laboratories Department, Faculty of Applied Medical Sciences, Taif University, Saudi Arabia \\ ${ }_{2}^{2}$ Medical Microbiology and Immunology Department, Faculty of Medicine, Menoufia University, Egypt \\ ${ }^{3}$ Medical Biochemistry Department, Faculty of Medicine, Zagazig University, Egypt \\ ${ }_{5}^{4}$ Medical Microbiology and Immunology Department, Faculty of Medicine, Zagazig University, Egypt \\ ${ }^{5}$ Medical Microbiology and Immunology Department Faculty of pharmacy, Taif University, Saudi Arabia \\ ${ }^{6}$ Clinical Pathology Department, Faculty of Medicine, Menoufia University, Egypt
}

\begin{abstract}
Introduction: Methicillin-resistant S. aureus (MRSA) is one of the most important organisms causing hospital-acquired infections worldwide. Molecular analysis of MRSA strains from Taif, Saudi Arabia, had not been previously done. Phenotypic and molecular characteristics of MRSA isolated from Taif hospitals were investigated.

Methodology: This study involved S. aureus strains isolated from different clinical samples from Taif hospitals. MRSA strains were identified and antimicrobial susceptibility profiles were determined. Multiplex polymerase chain reaction (PCR) was used to identify $S$. aureus-specific sequence, mecA genes, and type of staphylococcal cassette chromosome mec (SCCmec). MRSA strains were typed using coagulase gene polymorphism.

Results: In total, 390 strains of $S$. aureus were isolated, and 58 MRSA strains - 40 hospital-acquired MRSA (HA-MRSA) and 18 community-acquired MRSA (CA-MRSA) - were detected. HA-MRSA strains included three SCCmec types, while CA-MRSA strains included two SCCmec types. PCR amplification and restriction of the coagulase gene of the 58 MRSA isolates showed nine different patterns, while three strains were non-typable. HA-MRSA strains showed seven distinct restriction fragment length polymorphism (RFLP) patterns; the most frequent was pattern 2 (15 isolates), followed by patterns 1 and 4 (5 isolates each). CA-MRSA showed five RFLP patterns; the most frequent was pattern 3 ( 7 isolates) followed by pattern 8 (6 isolates).

Conclusions: HA-MRSA strains were more common than CA-MRSA strains. SCCmec typing and coagulase gene polymorphism analysis may be useful methods for studying clonal relatedness of isolates and for discriminating between HA-MRSA and CA-MRSA.
\end{abstract}

Key words: HA-MRSA; CA-MRSA; SCCmec; mecA gene.

J Infect Dev Ctries 2015; 9(3):298-303. doi:10.3855/jidc.5954

(Received 24 September 2014 - Accepted 23 November 2014)

Copyright $@ 2015$ Eed et al. This is an open-access article distributed under the Creative Commons Attribution License, which permits unrestricted use, distribution, and reproduction in any medium, provided the original work is properly cited.

\section{Introduction}

Healthcare-associated infections (HAIs) are considered among the most prevalent complications of hospital care [1]. Staphylococcus aureus (S. aureus) is responsible for a wide spectrum of infections ranging from superficial skin infections to fatal invasive infectious diseases including pneumonia, septicemia, and endocarditis [2]. Methicillin-resistant S. aureus (MRSA) was first reported in the early 1960s and has become a major nosocomial pathogen worldwide [3]. In the Gulf region, MRSA has been considered to be one of the most prevalent pathogens by Gulf Corporation Council (GCC) countries in the last two decades [4].

Methicillin resistance results from introduction of staphylococcal cassette chromosome mec (SCCmec) into methicillin-susceptible S. aureus strains. SCCmec is a genomic island that contains the antibiotic resistance gene mecA [5]. SCCmec also contains $c c r A$ and $c c r B$; both genes encode recombinases that mediate the site-specific integration and excision of the SCCmec element from the $S$. aureus chromosome [6,7]. The mecA gene codes for a variant type penicillin-binding protein (PBP2a), which has a lower affinity for the $\beta$-lactam antibiotics because its active site does not bind methicillin or other $\beta$-lactam antibiotics. Therefore, the transpeptidation reaction required for peptidoglycan cross-linking will continue, enabling cell wall synthesis [8]. Seven main types of SCCmec (type I to VII) are recognized. Types IV, V, VI, and VII code for $\beta$-lactam antibiotic resistance only, while SCCmec I, II, and III cause resistance to 
multiple classes of antibiotics, due to additional integrated drug resistance genes [9].

There are two major strains of MRSA: hospitalacquired MRSA (HA-MRSA) and communityacquired MRSA (CA-MRSA). They have been proven to be genetically distinct with respect to the SCCmec type; most HA-MRSA strains carry one of three types of SCCmec (types I, II, or III). On the other hand, CAMRSA strains carry mainly SCCmec type IV and occasionally type V [10].

In Saudi Arabia, few studies have been conducted to investigate the epidemiology of MRSA in the community or in hospitals. In 1991, a study showed that Saudi and British strains differed in their sensitivity patterns to antibiotics [11]. In Riyadh, it was found that overcrowding of patients and inadequate infection control measures were the major risk factors for MRSA acquisition [12]. In Abha (south region of Saudi Arabia), MRSA accounted for 5.1\% of hospital and $18.3 \%$ of non-hospital isolates [13]. To our knowledge, molecular analysis of MRSA strains in Taif area of Saudi Arabia has not been previously done. This study was conducted to investigate the phenotypic and molecular characteristics of MRSA isolates in Taif region, Saudi Arabia, and to compare CA-MRSA and HA-MRSA strains.

\section{Methodology}

Specimen collection and phenotypic testing

This study was conducted on MRSA strains collected form Taif hospitals between January 2013 and February 2014. Demographic, clinical, and laboratory data were collected after written consent was obtained. The clinical specimens of MRSA isolates included sputum, pus, pharyngeal swabs, urine, blood, and peritoneal fluid. The study proposal was approved by the Faculty of Applied Medical Sciences Ethics Committee of Taif University.

\section{Identification and antibiogram of $S$. aureus}

Initial screening and identification of $S$. aureus were performed according to the standard laboratory protocols. Isolates were further confirmed by detection of staphylococcal-specific genes by polymerase chain reaction (PCR). Antibiotic susceptibility profiles were determined and interpreted according to the guidelines of the Clinical and Laboratory Standards Institute (CLSI).

Methicillin resistance was tested by standard oxacillin salt agar screening plate procedure and cefoxitin susceptibility as indicated by the CLSI. Methicillin-resistant and methicillin-sensitive strains were included as controls [14]. Methicillin resistance was further confirmed by the detection of the mecA gene by the PCR method.

\section{DNA extraction}

Bacterial DNA was extracted by PrepMan Ultra Sample Preparation Reagent (Applied Biosystems, Foster city, USA) according to the manufacturer's instructions. Briefly, $1 \mathrm{~mL}$ of overnight lysogeny LB broth culture was centrifuged for 2 minutes. The pellet was re-suspended in phosphate buffer saline, then centrifuged again and the supernatant was discarded. PrepMan Ultra Sample Preparation Reagent $(100 \mu \mathrm{L})$ was added. The tubes were placed in a heat block at $100^{\circ} \mathrm{C}$ for 10 minutes. The tubes were allowed to cool to room temperature and centrifuged at the highest speed for 2 minutes. Then, $50 \mu \mathrm{L}$ of the supernatant was stored at $-20^{\circ} \mathrm{C}$ and $5 \mu \mathrm{L}$ was used for each PCR assay.

Detection of $S$. aureus-specific sequence gene and mecA gene

Multiplex PCR was used to amplify both the $S$. aureus-specific (107 bp) and mecA (532 bp) genes. The mecA gene was amplified using primers 5'- AAA ATC GAT GGT AAA GGT TGGC - 3' and 5'- AGT TCT GCA GTA CCG GAT TTG C-3'. The S. aureusspecific gene was amplified using primers 5'- AAT CTT TGT CGG TAC ACG ATA TTC TTC ACG -3' and 5'- CGT AAT GAG ATT TCA GTA GAT AAT ACA ACA -3'.The PCR reaction mixture and cycling conditions were done using the method of Martineau et al. [15].

\section{Staphylococcal cassette chromosome mec typing}

SCCmec typing was done by the multiplex PCR method [16]. The multiplex PCR included eight loci (A through $\mathrm{H}$ ) selected on the basis of the previously described mec element sequences. The multiplex PCR was performed using the GeneAmp PCR kit (Applied Biosystems).

PCR-restriction fragment length polymorphism (RFLP) typing of MRSA

MRSA strains were typed using coagulase gene polymorphism. The 3 ' end region of the coagulase gene was amplified by PCR as previously described by Lawrence et al.[17]. PCR amplification of the variable region of the coagulase gene was performed using primers COAG2-5'CGAGACCAAGA TTCAACAAG3' and COAG35'AAAGAAAACCACTCACATCA3'. 
Table 1. Clinical sources of the isolated MRSA andMSSA strains

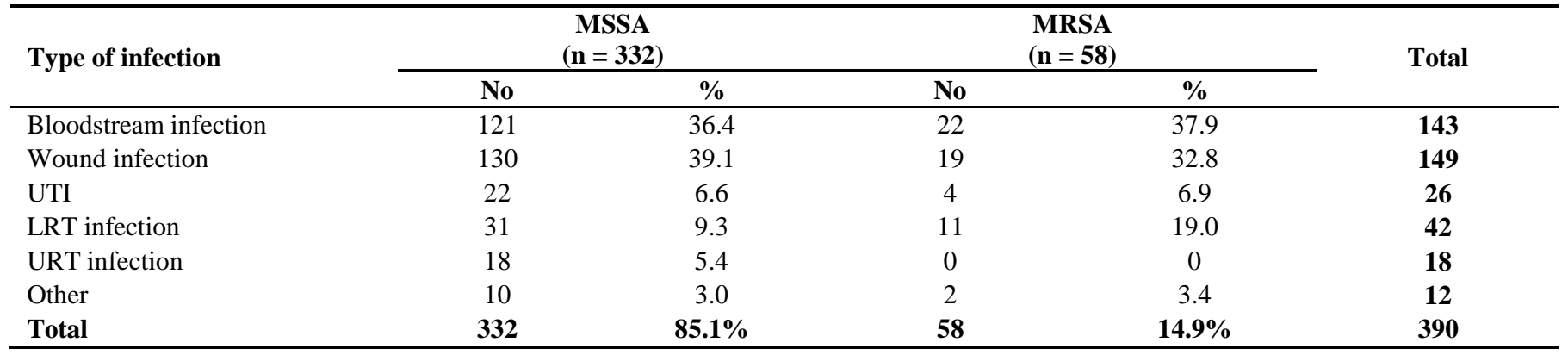

Table 2. Characterization of HA-MRSA strains, SCCmec type, PCR-RFLP of coa and mecA genes

\begin{tabular}{|c|c|c|c|c|c|}
\hline Strain no. & Source & SCCmec type & mec $\mathbf{A}$ & coa-RFLP profile (in bp) & Antibiotic resistance pattern \\
\hline MRSA1 & Wound swab & $\mathrm{I}$ & $+/+$ & (2) 81,567 & (2) PEN, OX, CEF, GN, TET, TS, RF \\
\hline MRSA2 & Wound swab & I & $+1+$ & (2) 81,567 & (2) PEN, OX, CEF, GN, TET, TS, RF \\
\hline MRSA3 & Blood & III & $+1+$ & (4) 234,486 & (1) PEN, OX, CEF, MU, ERY, CHL, TET, TS, CIP \\
\hline MRSA4 & Urine & I & $+1+$ & (1) 324,405 & (2) PEN, OX, CEF, GN, TET, TS, RF \\
\hline MRSA5 & Wound swab & III & $+/+$ & (9) $81,230,480$ & (1) PEN, OX, CEF, MU, ERY, CHL, TET, TS, CIP \\
\hline MRSA6 & Wound swab & I & $+1+$ & (2) 81,567 & (4) PEN, OX, CEF, GN, TS, RF \\
\hline MRSA7 & Blood & I & $+1+$ & (2) 81,567 & (6) PEN, OX, CEF, GN, ERY, TET, TS, CIP \\
\hline MRSA8 & Wound swab & I & $+1+$ & (2) 81,567 & (10) PEN, OX, CEF, ERY, CHL, TET, TS, CIP \\
\hline MRSA9 & Eye swab & I & $+/+$ & (2) 81,567 & (2) PEN, OX, CEF, GN, TET, TS, RF, TZP \\
\hline MRSA10 & Blood & III & $+1+$ & (9) $81,230,480$ & (6) PEN, OX, CEF, GN, ERY, TET, TS, CIP \\
\hline MRSA11 & Urine & III & $+1+$ & (4) 234,486 & (10) PEN, OX, CEF, ERY, CHL, TET, TS, CIP \\
\hline MRSA12 & Blood & III & $+1+$ & (4) 234,486 & (10) PEN, OX, CEF, ERY, CHL, TET, TS, CIP \\
\hline MRSA13 & Wound swab & III & $+1+$ & (4) 234,486 & (11) PEN, OX, CEF, GN, ERY, CHL, TET \\
\hline MRSA14 & Urine & I & $+1+$ & (1) 324,405 & (2) PEN, OX, CEF, GN, TET, TS, RF TZP \\
\hline MRSA15 & Wound swab & III & $+1+$ & (9) $81,230,480$ & (9) PEN, OX, CEF, GN, ERY, TET, TS \\
\hline MRSA16 & Blood & III & $+1+$ & (9) $81,230,480$ & (12) PEN, OX, CEF, GN, ERY, TET, TS \\
\hline MRSA17 & Wound swab & $\mathrm{I}$ & $+1+$ & (2) 81,567 & (4) PEN, OX, CEF, GN, TS, RF \\
\hline MRSA18 & Blood & II & $+1+$ & (6) $81,146,178,340$ & (12) PEN, OX, CEF, GN, ERY, TET, TS \\
\hline MRSA19 & Blood & II & $+1+$ & (7) $81,234,405$ & (1) PEN, OX, CEF, MU, ERY, CHL, TET, TS, CIP \\
\hline MRSA20 & Peritoneal fluid & II & $+1+$ & (7) $81,234,405$ & (3) PEN, OX, CEF, GN, MU,ERY, TET, TS, CIP \\
\hline MRSA21 & Blood & III & $+1+$ & None & (9) PEN, OX, CEF, GN, ERY, TET, TS \\
\hline MRSA22 & Sputum & $\mathrm{I}$ & $+1+$ & (2) 81,567 & (3) PEN, OX, CEF, GN, MU, ERY, TET, TS, CIP \\
\hline MRSA23 & Wound swab & III & $+1+$ & (9) $81,230,480$ & (9) PEN, OX, CEF, GN, ERY, TET, TS \\
\hline MRSA24 & Blood & III & $+1+$ & (9) $81,230,480$ & (12) PEN, OX, CEF, GN, ERY, TET, TS \\
\hline MRSA25 & Wound swab & I & $+/+$ & (2) 81,567 & (2) PEN, OX, CEF, GN, TET, TS, RF \\
\hline MRSA26 & Wound swab & I & $+1+$ & (2) 81,567 & (2) PEN, OX, CEF, GN, TET, TS, RF \\
\hline MRSA27 & Blood & II & $+1+$ & (7) $81,234,405$ & (5) PEN, OX, CEF, GN, ERY, CHL, TET, TS, CIP \\
\hline MRSA28 & Blood & II & $+1+$ & (1) 324,405 & (12) PEN, OX, CEF, GN, ERY, TET, TS \\
\hline MRSA29 & Blood & III & $+1+$ & (4) 234,486 & (5) PEN, OX, CEF, GN, ERY, CHL, TET, TS, CIP \\
\hline MRSA30 & Blood & III & $+/+$ & None & (2) PEN, OX, CEF, GN, TET, TS, RF \\
\hline MRSA31 & Sputum & II & $+/+$ & (7) $81,234,405$ & (8) PEN, OX, CEF, GN, SM, CHL, TET, CIP \\
\hline MRSA32 & Wound swab & I & $+/+$ & (2) 81,567 & (2) PEN, OX, CEF, GN, TET, TS, RF, TZP \\
\hline MRSA33 & Sputum & I & $+1+$ & (2) 81,567 & (4) PEN, OX, CEF, GN, TS, RF \\
\hline MRSA34 & Urine & III & $+1+$ & (9) $81,230,480$ & (7) PEN, OX, CEF, GN, MU, ERY, TET, TS, RF \\
\hline MRSA35 & Sputum & $\mathrm{I}$ & $+1+$ & (2) 81,567 & (9) PEN, OX, CEF, GN, ERY, TET, TS \\
\hline MRSA36 & Wound swab & I & $+/+$ & (2) 81,567 & (3) PEN, OX, CEF, GN, MU, ERY, TET, TS, CIP \\
\hline MRSA37 & Blood & III & $+1+$ & (8) $162,230,324$ & (5) PEN, OX, CEF, GN, ERY, CHL, TET, TS, CIP \\
\hline MRSA38 & Wound swab & II & $+1+$ & (1) 324,405 & (5) PEN, OX, CEF, GN, ERY, CHL, TET, TS, CIP \\
\hline MRSA39 & Sputum & I & $+1+$ & (2) 81,567 & (2) PEN, OX, CEF, GN, TET, TS, RF \\
\hline MRSA40 & Sputum & I & $+1+$ & (1) 324,405 & (4) PEN, OX, CEF, GN, TS, RF \\
\hline
\end{tabular}

PEN: penicillin, OX: oxacillin, CEF: cefoxitin, GN: gentamicin, SM: streptomycin, ERY: erythromycin, CHL: chloramphenicol, TET: tetracycline, TS: trimethoprim, RF: rifamipicin, CIP: ciprofloxacin, TZP: piperacillin-tazobactam and MU: mupirocin. 
After amplification, $15 \mu \mathrm{L}$ of the PCR products was digested for 15 minutes with 6 IU of restriction endonuclease Alul (Fermentas, Sunderland UK) according to the manufacturer's protocol. Restriction digest fragments were separated by $2 \%$ agarose electrophoresis [18].

\section{Results}

The studied strains included $390 \mathrm{~S}$. aureus that were isolated from male $(\mathrm{n}=249)$ and female $(\mathrm{n}=$ 141) Saudi $(\mathrm{n}=334)$ and non-Saudi $(\mathrm{n}=56)$ patients (Table 1). Of them, 58 MRSA strains (40 HA-MRSA and 18 CA-MRSA) were detected and characterized. All the isolated $S$. aureus strains were susceptible to vancomycin and linezolid. All the MRSA isolates were resistant to penicillin, oxacillin, and cefoxitin. Various resistance patterns were demonstrated for the other antibiotics (Table 2). HA-MRSA strains were more resistant than CA-MRSA to the most tested non$\beta$-lactam antibiotics, including ciprofloxacin, gentamicin, tetracycline, chloramphenicol, and tetracycline.

HA-MRSA strains $(n=40)$ were grouped into three SCCmec types: type I $(\mathrm{n}=18)$, type II $(\mathrm{n}=7)$, and type III $(\mathrm{n}=15)$. CA-MRSA strains were grouped into two SCCmec types: type IV $(\mathrm{n}=11)$ and type V $(\mathrm{n}=6)$. However, one isolate was non-typeable using the multiplex SCCmec (Tables 2 and 3).PCR products of coagulase gene amplification ranged from 648 to $810 \mathrm{bp}$. The restriction DNA digests were estimated by comparison with a 100-bp DNA marker. PCR amplification and restriction of the coagulase gene of the 58 MRSA isolates showed nine different patterns (Tables 2 and 3), while three strains were non-typable. HA-MRSA strains showed seven distinct RFLP patterns; the most frequent was pattern 2 (15 isolates, $37.5 \%$ ), followed by patterns 1 and 4 (5 isolates each, $12.5 \%)$. CA-MRSA showed five RFLP patterns; the most frequent was pattern 3 (7 isolates, 38.9\%) followed by pattern 8 (6 isolates, $33.3 \%$ ).

\section{Discussion}

MRSA is an increasing problem and its burden continues to rise in healthcare facilities [19]. Rising colonization rates lead to increased infection rates in hospitals. This leads to significant increased morbidity and mortality rates due to invasive MRSA infection $[20,21]$. In our study, MRSA accounted for $14.8 \%$ ( $\mathrm{n}=$ 58 ) of $390 \mathrm{~S}$. aureus strains. This rate is similar to a previous report in New South Wales, Australia [21]. However, a more recent report showed a much lower rate [22]. In Saudi Arabia, an earlier study demonstrated that MRSA accounted for $7.5 \%$ of all $S$. aureus isolates in a three-year epidemiological study in Jeddah [23].

Antibiogram allows quick and early recognition of a previously defined epidemic strain in a particular hospital setting. In our study, the antibiotic resistance patterns were similar to those previously reported in Korea by Goh et al. [24]. Multiplex PCR SCCmec typing of 40 MRSA isolates obtained from clinical cultures showed that 18 isolates were SCCmec type I, 15 isolates were type III, and 7 isolates were type II. Among the CA-MRSA strains, the most common

Table 3. Characterization CA-MRSA strains, SCCmec type, PCR-RFLP of coa and mecA genes

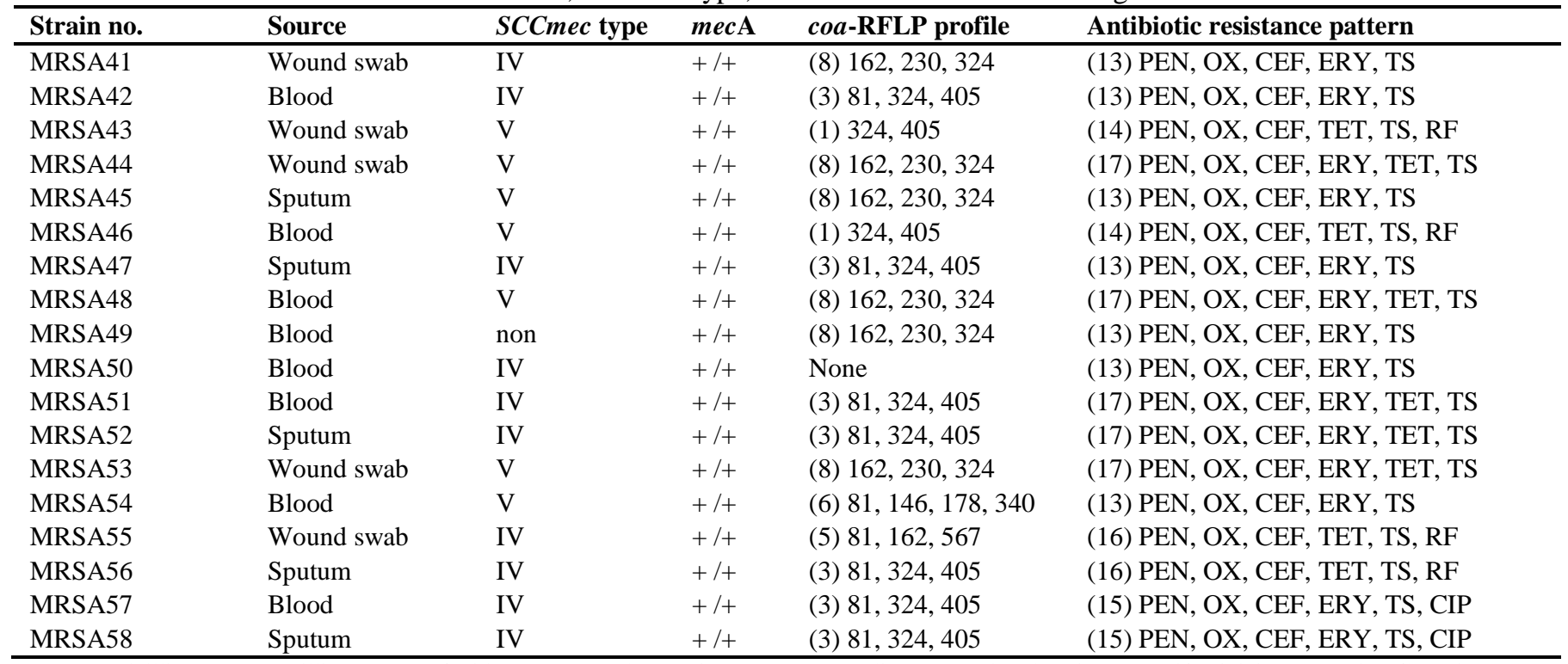

PEN: penicillin, OX: oxacillin, CEF: cefoxitin, ERY: erythromycin, TET: tetracycline, TS: trimethoprim, RF: rifamipicin, CIP: ciprofloxacin, TZP: piperacillin-tazobactam and MU: mupirocin 
SCCmec type was type IV (10 isolates) followed by type V (7 isolates), a finding similar to that of Dejing et al. [25]. These results suggest that CA- and HAMRSA are likely to coexist in our hospitals, as previously reported in the United States [26].

In this study, the SCCmec types correlated well with the major antibiotic susceptibility patterns. Although previous studies have shown that SCCmec types IV and V, which are CA-MRSA strains, were generally susceptible to non $\beta$-lactam antibiotics and more susceptible to antibiotics than were HA-MRSA strains $[3,20]$. Moreover, our results demonstrated that SCCmec type IV (2 isolates) and SCCmec type V (3 isolates) were resistant to many non- $\beta$-lactam antibiotics. This finding was noted in previous studies, suggesting that SCCmec types IV and V strains can acquire resistance to non- $\beta$-lactam antibiotics in order to tolerate hospital environments and excessive antibiotic exposure [27].

Pulsed-field gel electrophoresis (PFGE) is considered the most discriminatory and reliable method of typing. However, it is more complex and time consuming. Furthermore, it was not available in our laboratory. PCR-RFLP typing of the coagulase gene $(c o a)$ can be used for $S$. aureus typing on the basis of DNA variation within the 3 ' end coding region of the gene [28]. Since the isolation of the staphylococcal coa gene in 1987, AluI and, to a lesser extent, Hue,III-RFLP digestion of the coa gene has been extensively used for differentiation of MRSA strains with excellent correlation between RFLP patterns and multi-locus enzyme electrophoresis [2]. PCR amplification and restriction of the coa gene of the studied 58 MRSA isolates generated nine different patterns. Four RFLP patterns $(2,4,7,9)$ were detected only in HA-MRSA, and two RFLP patterns $(3,5)$ were detected only in CA-MRSA, while other RFLP patterns $(1,6,8)$ were shared by both types of MRSA. Our results are in accordance with those of Kobayashi et al. [29], who found that MRSA and MSSA were classified into 6 and 12 RFLP patterns, respectively, with 5 patterns detected frequently in both MRSA and MSSA. Walker et al. [18] showed that AluI digestion of the coa gene PCR products of 356 MRSA strains yielded 13 different RFLP patterns. In contrast to our results, Lawrence et al. [17] isolated MRSA strains from various hospitals and showed that the strains were closely related and that $91.8 \%$ of the isolated strains had a unique RFLP pattern when analyzed by coagulase gene typing.

\section{Conclusions}

In conclusion, HA-MRSA strains were more common than CA-MRSA strains in Taif. Coagulase gene polymorphism requires only small quantities of DNA, and isolates can be compared by both the number of PCR products and the size of their AluI restriction fragments. MRSA RFLP analysis yielded nine distinct types, and HA-MRSA and CA-MRSA were discriminated to a great extent. Coagulase gene polymorphism is an easy and accurate method for studying clonal relatedness of isolates.

\section{Acknowledgements}

This study was funded by the Academic Research Center at Taif University, project number 1-434-2565.

\section{References}

1. Baird VL, Hawly R (2000) Methicillin-resistant Staphylococcus aureus (MRSA): there a need to change clinical practice? Inten Crit Care Nurs 16: 357-366.

2. Christine KH, Christine MS, Wolfgang H, Timothy WM (2011) Molecular epidemiology of methicillin-resistant and methicillin-susceptible Staphylococcus aureus isolated from the eye. Current Eye Res 36: 94-102.

3. Abbasi K (1998) Facing the microbial threat. BMU 317: 620626.

4. Mahmoud A, Balkhy H (2012) The prevalence of antimicrobial resistance in clinical isolates from Gulf Corporation Council countries. Antimicrob Resist Infect Control 1: 26-28.

5. Hartman B, Tomasz A (1981) Altered penicillin-binding proteins in methicillin-resistant strains of Staphylococcus aureus. Antimicrob Agents Chemother 19: 726-35.

6. Pantosti A, Sanchini A, Monaco M (2007) Mechanisms of antibiotic resistance in Staphylococcus aureus. Future Microbiol 2: 323-334.

7. Biswajit B, Gautam KR, Shibendu B (2012) Methicillinresistant Staphylococcus aureus: A Brief review. Int Res J of Bio Sci 1: 65-71.

8. Lowy FD (2003) Antimicrobial resistance: the example of Staphylococcus aureus. J Clin Invest 111: 1265-1273.

9. Oliveira DC, Tomasz A, de Lencastre H (2001) The evolution of pandemic clones of methicillin-resistant Staphylococcus aureus: identification of two ancestral genetic backgrounds and the associated mec elements. Microb Drug Resist 7: 349361.

10. Vandenesch F, Naimi E, Enright MC (2003) Communityacquired methicillin-resistant Staphylococcus aureus carrying Panton-Valentine leukocidin genes: worldwild emergence. Emerg Infect Dis 9: 978-984.

11. Bukharie HA, Abdelhadi MS, Saeed IA, Rubaish AM, Larbi EB (2001) Emergence of methicillin-resistant Staphylococcus aureus as a community pathogen. Diagn Microbiol Infect Dis 40: $1-4$.

12. Haddad Q, Sobayo EI, Basit OB, Rotimi VO (1993) Outbreak of methicillin-resistant Staphylococcus aureus in a neonatal intensive care unit. J Hosp Infect 23: 211-222.

13. Alghaithy AA, Bilal NE, Gedebou M, Weily AH (2000) Nasal carriage and antibiotic resistance of Staphylococcus 
aureus isolates from hospital and non-hospital personnel in Abha, Saudi Arabia. Trans R Soc Trop Med Hyg 94: 504-507.

14. Kotilainen P, Routamaa M, Peltonen R, Oksi J, Rintala, Meurman E, Lehtonen OP, Eerola E, Salmenlinna S, VuopioVarkila J, Rossi T (2003) Elimination of epidemic methicillin-resistant Staphylococcus aureus from a university hospital and district institutions. Finland Emerg Infect Dis 9: 169-175.

15. Martineau F, Picard FJ, Lansac N (2001) Correlation between the mecA gene determined by multiplex PCR assays and the disc diffusion susceptibility patterns of Staphylococcus aureus. Chemother 44: 231-238.

16. Duarte CO, Hermínia L (2002) Multiplex PCR strategy for rapid identification of structural types and variants of the mec element in methicillin-resistant Staphylococcus aureus. Antimicrob Agent Chemother 46: 2155-2161.

17. Lawrence C, Cosseron M, Mimoz O (1996) Use of the coagulase gene typing method for the detection of carriers of methicillin-resistant Staphylococcus aureus. J Antimicrob Chemother 37: 687-696.

18. Walker J, Borrow R, Edwards-J V, Oppengheim BA, Fox AL (1998) Epidemiological characterization of methicillinresistant Staphylococcus aureus isolated in the North West of England by protein A (spa) and coagulase (coa) gene polymorphisms. Epidemiol Infect 121: 507-514.

19. Van Hal SJ, Stark D, Lockwood B, Marriott D Harkness J (2007) Methicillin-resistant Staphylococcus aureus (MRSA) detection: Comparison of two molecular methods (IDI-MRSA PCR assay and genotype MRSA direct PCR Assay) with three selective MRSA agars (MRSA ID, MRSASelect, and CHROM agar MRSA) for use with infection control swabs. J Clin Microbiol 45: 2486-2490.

20. Lodise TP, McKinnon PS (2005) Clinical and economic impact of methicillin resistance in patients with Staphylococcus aureus bacteremia. Diagn Microbiol Infect Dis 52: 113-122.

21. Nimmo, GR, Coombs JC, Pearson FG, O'Brien KJ, Christiansen JD, Gosbell IB, Collignon P, McLaws M (2006) Methicillin-resistant Staphylococcus aureus in the Australian community: an evolving epidemic Med J 184: 384-388.

22. James WS, Gina KM, Charles LJ (2010) Comparison of the BD GeneOhm Methicillin-Resistant Staphylococcus aureus (MRSA) PCR assay to culture by use of BBL CHROMagar MRSA for detection of MRSA in nasal surveillance cultures from intensive care unit patient. J Clin Microbiol 32: 407-415.
23. Zaman R, Dibb WL (1994) Methicillin resistant Staphylococcus aureus (MRSA) isolated in Saudi Arabia: Epidemiology and antimicrobial resistance patterns. J Hosp Infect 26: 297-300.

24. Goh S, Byrne SK, Zhang JL, Chow AW (1992) Molecular typing of Staphylococcus aureus on the basis of coagulase gene polymorphism. J Clin Microbiol 30: 1642-1645.

25. Dejing W, Qun W, Yonghong Y, Wenjing G, Qiang W, Sangjie Y, Kaihu Y, Lin Y, Xuzhuang S (2010) Epidemiology and molecular characteristics of communityassociated methicillin-resistant and Methicillin-susceptible Staphylococcus aureus from skin/soft tissue infections in a children's hospital in Beijing, China. Diagnostic Microbiol Infect Dis 67: 1-8.

26. Charlebois ED, Bangsberg DR, Moss NJ, Moore MR, Moss AR, Chambers HF, Perdreau RF (2002) Population-based community prevalence of methicillin-resistant Staphylococcus aureus in the urban poor of San Francisco. Clin Infect Dis 34: 425-433.

27. Barham F, Mohammad E, Marzieh A, Gwedolyn G, Mohammad MF (2009) Molecular characterization of MRSA form teaching hospital in Tehran. Jpn J Infect Dis 62: 309322.

28. Keiko I, Naofumi T, Jun I, Kunimoto H (2007) Usefulness of PCR-restriction fragment length polymorphism typing of the coagulase gene to discriminate Arbekacin-resistant methicillin-resistant Staphylococcus aureus strains. J Clin Microbiol 45: 607-609.

29. Kobayashi N, Taniguchi K, Kojima K (1995) Analysis of methicillin-resistant and methicillin-susceptible Staphylococcus aureus by a molecular typing method based on coagulase gene polymorphisms. Epidemiol Infect 115: $419-426$.

\section{Corresponding author}

Emad M. Eed

Medical Laboratories Department

Faculty of Applied Medical Sciences, Taif University

55 Abo Bakr St, Taif, Saudi Arabia

Phone: +966582765258

Email: Dremadeed@yahoo.com

Conflict of interests: No conflict of interests is declared. 